\title{
Factors associated with red blood cell transfusion among hospitalized patients: a cross-sectional study
}

\author{
Peiwen ZHANG ${ }^{1}$, Dandan $\mathrm{XU}^{1}$, Xinhan ZHANG ${ }^{2}$, Mengyin $\mathrm{WU}^{2}$, Xuecheng $\mathrm{YAO}^{2}$, Dawei CUI ${ }^{1}$, Jue XIE ${ }^{1 \bowtie}$ \\ ${ }^{I}$ Department of Blood Transfusion, the First Affiliated Hospital, Zhejiang University School of Medicine, Hangzhou 310003, China \\ ${ }^{2}$ Department of Epidemiology and Biostatistics, Zhejiang University School of Public Health, Hangzhou 310058, China
}

Red blood cell (RBC) transfusion is a clinically effective therapy in anemia, for example in patients with malignancies (Shander et al., 2020), bleeding (Odutayo et al., 2017), and preoperative anemia (Padmanabhan et al., 2019). The past few decades have witnessed a shortage of blood for transfusion due to limited health insurance coverage for blood use and the rapid expansion of hospitals (Chen et al., 2011; Shi et al., 2014). Blood donation levels may easily be affected by general changes in the environment, policy, major events such as disasters, and public sentiment (Hu et al., 2019). Meanwhile, the transfusion of allogeneic RBC is a double-edged sword, increasing the possibility of infectious and immunological complications, and also leading to higher morbidity and mortality after transfusion (Frank et al., 2012). Considering that the continual shortfall has been increasingly prominent, identifying the factors associated with $\mathrm{RBC}$ transfusion could help blood transfusion departments to improve their supply of blood products as well as their inventory management (O'Donnell et al., 2018).

Previous studies have considered blood shortages at either the hospital level or the patient level. Here, we analyzed the imbalance from both these perspectives, based on a large amount of data collected from the First Affiliated Hospital (Zhejiang University School of Medicine, Hangzhou, China). For this study, we collected data on patients' $\mathrm{RBC}$ requirements from January 1st, 2013 to December 31st, 2020. The daily total applied units of RBC were cumulative for all

$\triangle$ Jue XIE, zyyyxj2011@zju.edu.cn

(D) Jue XIE, https://orcid.org/0000-0002-4270-671X

Received June 3, 2021; Revision accepted Sept. 17 2021; Crosschecked Nov. 22, 2021

(C) Zhejiang University Press 2021 patient requirements during this period. We established a time series based on total weekly RBC applications for the entire hospital from 2013 to 2020 (Fig. 1a).

We conducted a time-series analysis using the triple exponential smoothing method to decompose the factors from the hospital inventory which affected the amount of RBC given from 2013 to 2019 (Figs. 1b-1e). Because of the impact of the coronavirus disease 2019 (COVID-19) in early 2020 (Xiang et al., 2020), there was a sizeable reduction in the total number of applications. Therefore, we did not include the data for this period in the analysis. Accordingly, the amounts of weekly RBC applications showed an obvious upward trend year by year, as well as a similar seasonality each year.

The basic principle of the exponential smoothing method is to give different weights to observed values of a time-series sequence. Compared with earlier data, more recent data will be given greater weight, which helps to eliminate the influence of noise and produce more reasonable and reliable models. The triple exponential smoothing method, which applies exponential smoothing three times, allowed us to incorporate seasonal effects into the model.

Holt-Winters multiplicative fitting and Holt-Winters additive fitting are the two main triple exponential smoothing methods. If a time series is stable, HoltWinters additive fitting is recommended; otherwise, Holt-Winters multiplicative fitting is preferred. Overall, we saw little difference between the two models in fitting the total weekly RBC applications. The horizontal parameter $(\alpha)$ was 0.072 vs. 0.097 , the trend parameter $(\beta)$ was 0 vs. 0 , and the seasonal parameter $(\gamma)$ was 0.454 vs. 0.383 (multiplicative vs. additive). Most donors in China are college students and migrant 
workers; thus during the summer college vacation and the traditional Chinese Spring Festival Holiday, the donation decreased dramatically. The amount peaked in August and September, decreased from October, and then gradually rose again in December.

Information about patients receiving $\mathrm{RBC}$ included the number of units applied, patient age (years), sex, and body mass index (BMI, $\mathrm{kg} / \mathrm{m}^{2}$ ), as well as the results of a blood test (hemoglobin (HGB), platelet (PLT), prothrombin time (PT), hematocrit (HCT), and alanine aminotransferase (ALT)), ABO type, usage classification, history of blood transfusion (Xiang et al., 2020), and hospital department. We used a binary logistic regression model to estimate the odds ratio (OR) and 95\% confidence interval (CI) for the effects of selected individual and seasonal variables on the number of transfusion units. The units of RBC given were categorized into a binary variable by the median of transfused RBC, which was 0 (for $<2$ units) or 1 (for $\geqslant 2$ units). We adjusted for all selected factors in the first model, including age (years), sex (male/female), BMI $\left(<18.5 \mathrm{~kg} / \mathrm{m}^{2}, 18.5-23.9 \mathrm{~kg} / \mathrm{m}^{2}, 24.0-27.9 \mathrm{~kg} / \mathrm{m}^{2}\right.$,

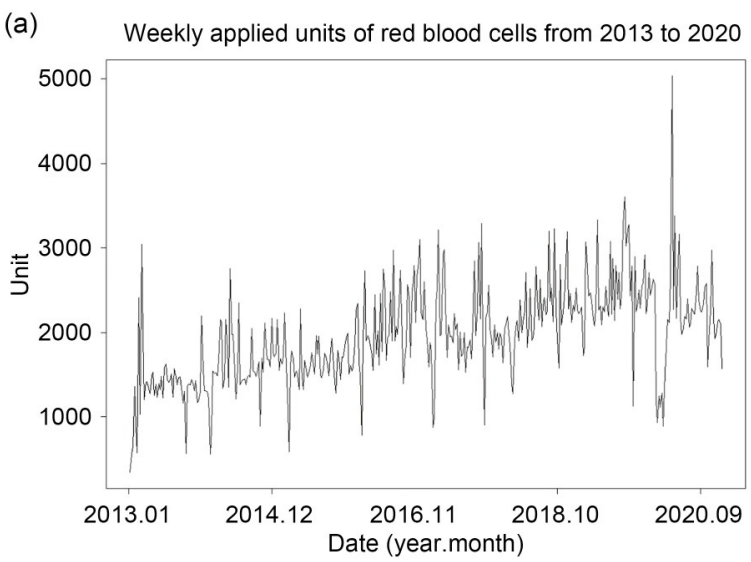

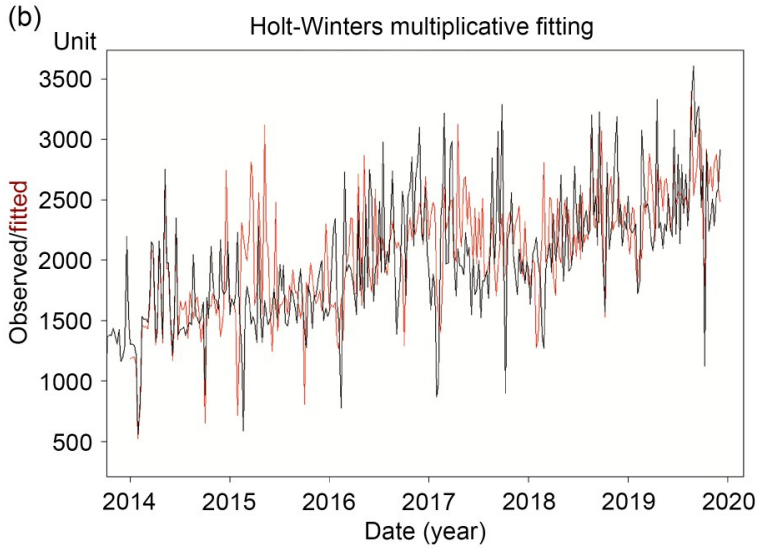

(d)

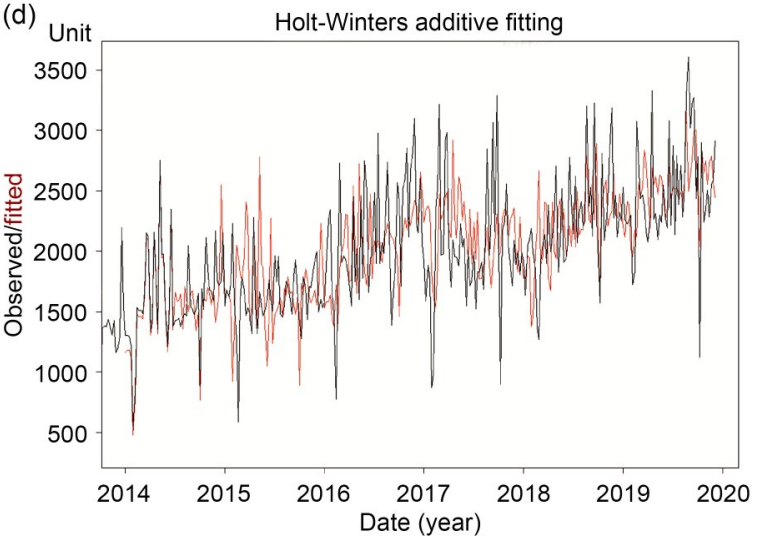

(c)

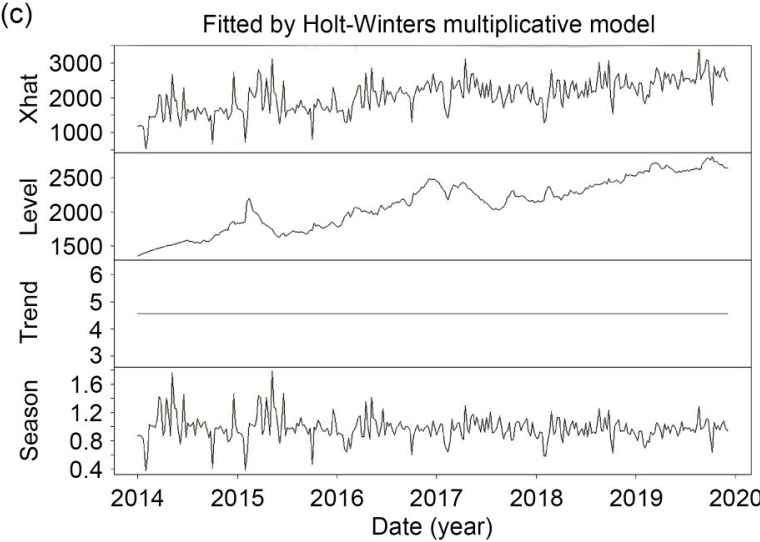

(e)

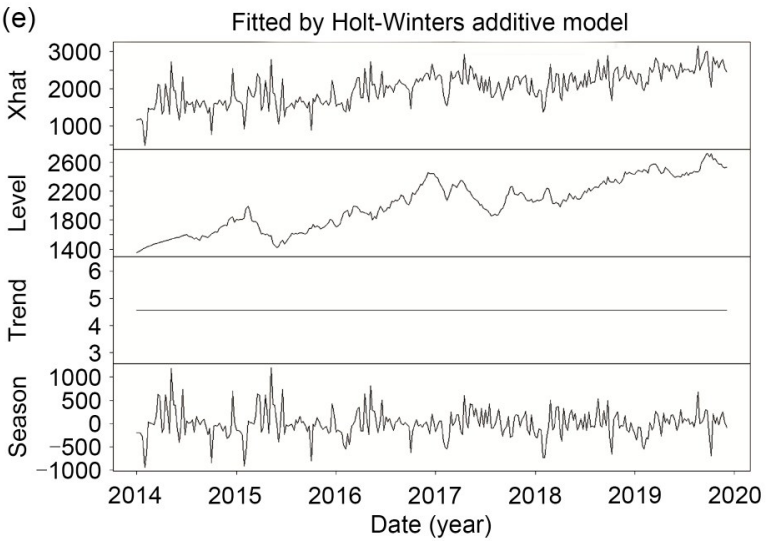




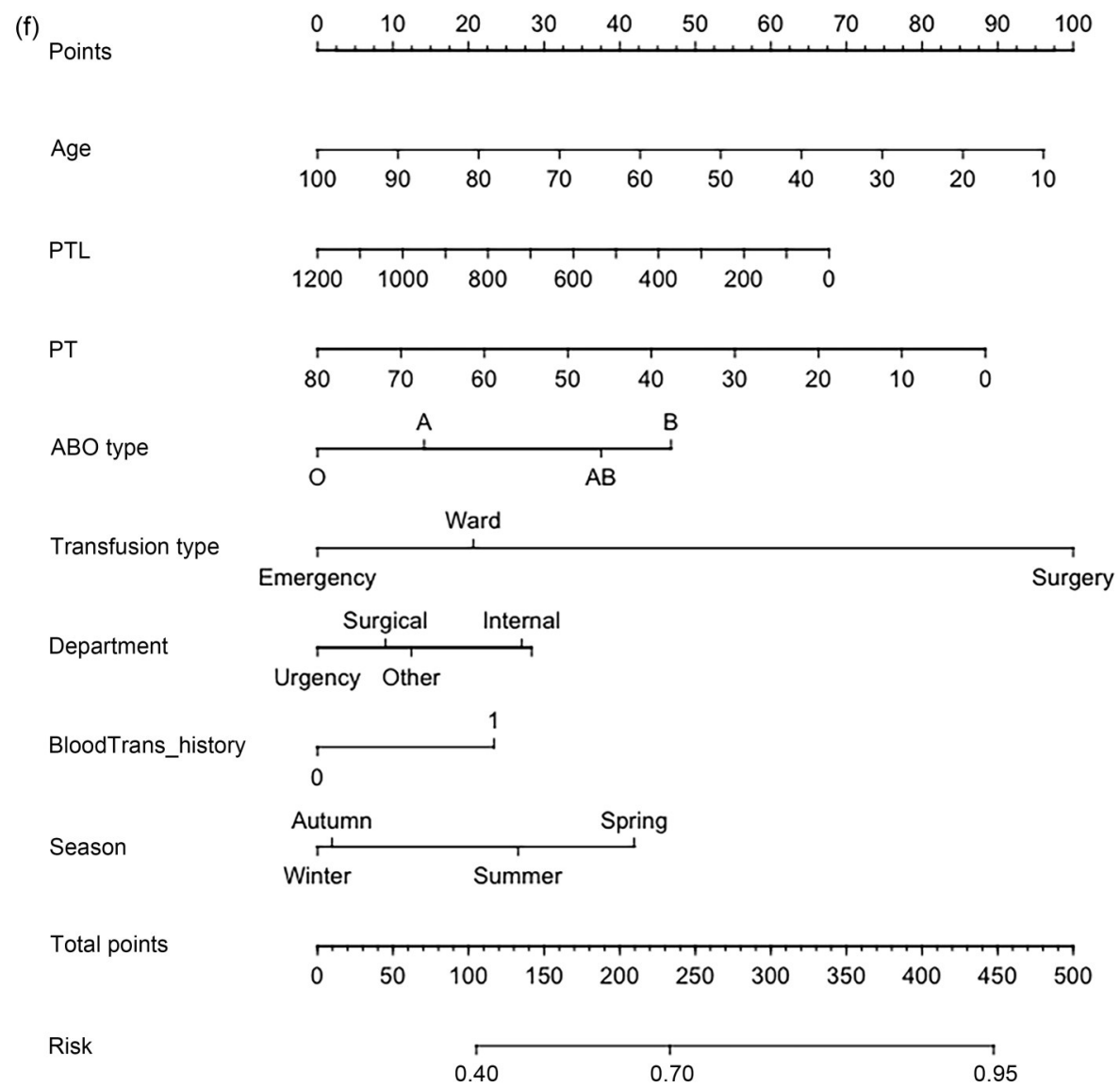

Fig. 1 Models derived from clinical blood transfusion data. (a) Time plot of the daily and weekly demands for RBC transfusion from Jan. 2013 to Dec. 2020. (b-e) Fitting graphs and component decomposition graphs produced with the Holt-Winters multiplicative and additive models: (b) fitting graph for the Holt-Winters multiplicative model; (c) component decomposition graph for the Holt-Winters multiplicative model; (d) fitting graph for the Holt-Winters additive model; (e) component decomposition graph for the Holt-Winters additive model. (f) Nomogram showing the risk of transfusing RBC below or beyond two units. RBC: red blood cell; PLT: platelet; PT: prothrombin time.

and $\left.\geqslant 28.0 \mathrm{~kg} / \mathrm{m}^{2}\right)$, HGB $(\mathrm{g} / \mathrm{L})$, PLT $\left(10^{9} \mathrm{~L}^{-1}\right)$, PT (s), HCT $(\%)$, ALT (U/L), ABO blood type, usage classification (surgery, ward, and emergency), history of blood transfusion (Yes/No), departments (surgical department, internal medicine department, obstetrics and gynecology department, emergency department, and others), and season (spring, summer, autumn, or winter). We then tried the second model, using backward step-wise binary logistic regression based on the first model. In addition, to assist in translation of the generated statistical model, we created a risk assessment nomogram as a graphic model representation (Fig. 1f). Subgroup analysis was performed to identify potential modifications by different seasons.

Patients receiving $\mathrm{RBC}$ transfusion were aged $(55.91 \pm 16.20)$ years and $56 \%$ male. Blood types A $(33.6 \%)$ and $\mathrm{O}(36.3 \%)$ were in greater demand.
Almost half of patients $(48.1 \%)$ received $\mathrm{RBC}$ in connection with surgery and $59.8 \%$ of them had a history of blood transfusion (Table S1).

We found that older age and higher PLT were related to less RBC transfusion. Patients with $\mathrm{AB}$ (OR (95\% CI): $1.26(1.04,1.54))$ or B (OR $(95 \%$ CI): 1.38 $(1.21,1.57))$ blood types were more likely to have $\mathrm{RBC}$ transfusions of $>2$ units compared to type A. Conversely, patients with type O (OR (95\% CI): 0.87 $(0.79,0.97))$ are unlikely to have $\mathrm{RBC}$ transfusions of $>2$ units. Patients who were having elective surgery (OR (95\% CI): $2.70(2.24,3.27))$ or were hospitalized (OR (95\% CI): 1.23 (1.07, 1.41)) needed more RBC transfusion compared to those in the emergency department. Also, having a history of blood transfusion was a risk factor for greater RBC need. Compared with the internal medicine department (OR (95\% CI): 2.23 
$(2.07,2.41))$, obstetrics and gynecology department (OR $(95 \%$ CI): $0.83(0.70,0.97))$ and emergency department (OR (95\% CI): $0.75(0.64,0.88))$ were less likely to use RBC transfusions over 2 units. In general, the amount of blood used in the internal medicine department was higher in autumn (Fig. S1). Furthermore, patients who received RBC in summer (OR $(95 \%$ CI): $0.72(0.66,0.79))$ were negatively correlated to higher units of RBC transfusion, while in winter and autumn (OR (95\% CI): $1.11(1.01,1.22))$, there was a positive correlation (Table S2).

In subgroup analysis, factors affecting the units of RBC transfusion varied across the four seasons (Table S3). For all four seasons, the transfusion demand for surgery was steadily higher than that for emergency events. In spring, male and O-type patients were less likely to have more units of RBC transfusion. Meanwhile, in the other three seasons, younger and B-type patients were associated with more $\mathrm{RBC}$ transfusion units. In summer, the HGB (OR (95\% CI): 0.99 (0.97, $1.00))$ and HCT (OR $(95 \% \mathrm{CI}): 1.04(1.00,1.09))$ of the patients were significantly related to the number of RBC transfusion units. A history of blood transfusion (OR $(95 \% \mathrm{CI})$ : $1.48(1.10,1.98))$ was also a risk factor for more RBC transfusion, but only in summer. In autumn, overweight and AB-type patients were positively associated with higher demand for RBC, and the need in the emergency department was less than that in the internal medicine department. There were fewer factors significantly related to RBC transfusion in winter than in the other three seasons.

In summary, we collected a substantial amount of data on RBC transfusion in a large general hospital in Zhejiang Province, China. We conducted a time-series analysis using the triple exponential smoothing method and found a seasonal trend in the demand for RBC. Then, we created a binary logistic model based on patients' selected factors as well as seasonal trends. Taken together, the results suggest that age, PLT, PT, ABO type, different usage classification, history of blood transfusion, and hospital department are the possible determinants, and that the effects of these factors vary seasonally. The units of RBC given are associated with seasonal trends in the blood inventory and overall state of patients in this large general hospital. Further studies involving more hospital-level indicators may give more insight into best practices in blood transfusion management.

\section{Materials and methods}

Detailed methods are provided in the electronic supplementary materials of this paper.

\section{Acknowledgments}

This work was supported by the National Natural Science Foundation of China (Nos. 91846103 and 81971994) and the Zhejiang Provincial Key Research and Development Program (No. 2020C03032), China.

\section{Author contributions}

Peiwen ZHANG wrote the manuscript. Dandan XU collected the data. Xinhan ZHANG analyzed the hospital data using the triple exponential smoothing method. Mengyin WU analyzed the patient data with binary logistic regression model. Xuecheng YAO made the nomogram graph. Dawei CUI adjusted the scope of research and coordinated the needs of all participants. Jue XIE contributed to the study design and was responsible for the integrity of the study and final manuscript. All authors have read and approved the final manuscript, and therefore, have full access to all the data in the study and take responsibility for the integrity and security of the data.

\section{Compliance with ethics guidelines}

Peiwen ZHANG, Dandan XU, Xinhan ZHANG, Mengyin WU, Xuecheng YAO, Dawei CUI, and Jue XIE declare that they have no conflict of interest.

All procedures followed were in accordance with the ethical standards of the responsible committee on human experimentation (the First Affiliate Hospital, Zhejiang University School of Medicine, Hangzhou, China) and with the Helsinki Declaration of 1975. Informed consent was obtained from all patients for being included in the study. Additional informed consent was obtained from all patients for whom identifying information is included in this article.

\section{References}

Chen XF, Chen CL, Zhang Y, et al., 2011. The effect of health insurance reform on the number of cataract surgeries in Chongqing, China. BMC Health Serv Res, 11:67. https://doi.org/10.1186/1472-6963-11-67

Frank SM, Savage WJ, Rothschild JA, et al., 2012. Variability in blood and blood component utilization as assessed by an anesthesia information management system. Anesthesiology, 117(1):99-106. https://doi.org/10.1097/ALN.0b013e318255e550

Hu W, Meng HD, Hu QY, et al., 2019. Blood donation from 2006 to 2015 in Zhejiang Province, China: annual consecutive cross-sectional studies. BMJ Open, 9:e023514. http://dx.doi.org/10.1136/bmjopen-2018-023514

O'Donnell TFX, Shean KE, Deery SE, et al., 2018. A preoperative risk score for transfusion in infrarenal endovascular aneurysm repair to avoid type and cross. J Vasc Surg, 
67(2):442-48

https://doi.org/10.1016/j.jvs.2017.05.108

Odutayo, A, Desborough MJR, Trivella M, et al., 2017. Restrictive versus liberal blood transfusion for gastrointestinal bleeding: a systematic review and meta-analysis of randomised controlled trials. Lancet Gastroenterol Hepatol, 2(5):354-360.

https://doi.org/10.1016/S2468-1253(17)30054-7

Padmanabhan H, Brookes MJ, Nevill AM, et al., 2019. Association between anemia and blood transfusion with long-term mortality after cardiac surgery. Ann Thorac Surg, 108(3):687-692.

https://doi.org/10.1016/j.athoracsur.2019.04.044

Shander A, Kaulfman M, Goognough LT, 2020. How I treat anemia in the perisurgical setting. Blood, 136(7):814-822. https://doi.org/10.1182/blood.2019003945

Shi L, Wang JX, Stevens L, et al., 2014. Blood safety and availability: continuing challenges in China's blood banking system. Transfusion, 54(2):471-482. https://doi.org/10.1111/trf.12273

Xiang WL, Cheng JJ, Wu LP, et al., 2020. Clinical characteristics and plasma antibody titer of patients with COVID-19 in Zhejiang, China. J Zhejiang Univ-Sci B (Biomed \& Biotechnol), 21(12):955-960.

https://doi.org/10.1631/jzus.B2000593

\section{Supplementary information}

Materials and methods; Tables S1-S3; Fig. S1 